\title{
OPEN Multispectral imaging detects gastritis consistently in mouse model and in humans
}

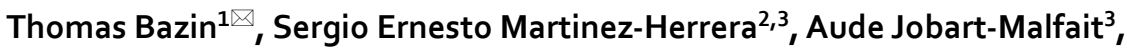
Yannick Benezeth ${ }^{4}$, Matthieu Boffety ${ }^{2}$, Catherine Julié5 ${ }^{5}$, Jean-François Emile ${ }^{5}$, Valérie Michel $^{6}$, François Goudail ${ }^{2}$, Eliette Touati ${ }^{6}$, Franck Marzani ${ }^{4}$ \& Dominique Lamarque ${ }^{1}$

Gastritis constitutes the initial step of the gastric carcinogenesis process. Gastritis diagnosis is based on histological examination of biopsies. Non-invasive real-time methods to detect mucosal inflammation are needed. Tissue optical properties modify reemitted light, i.e. the proportion of light that is emitted by a tissue after stimulation by a light flux. Analysis of light reemitted by gastric tissue could predict the inflammatory state. The aim of our study was to investigate a potential association between reemitted light and gastric tissue inflammation. We used two models and three multispectral analysis methods available on the marketplace. We used a mouse model of Helicobacter pylori infection and included patients undergoing gastric endoscopy. In mice, the reemitted light was measured using a spectrometer and a multispectral camera. We also exposed patient's gastric mucosa to specific wavelengths and analyzed reemitted light. In both mouse model and humans, modifications of reemitted light were observed around $560 \mathrm{~nm}, 600 \mathrm{~nm}$ and $640 \mathrm{~nm}$, associated with the presence of gastritis lesions. These results pave the way for the development of improved endoscopes in order to detect real-time gastritis without the need of biopsies. This would allow a better prevention of gastric cancer alongside with cost efficient endoscopies.
\end{abstract}

Chronic gastritis is the consequence of Helicobacter pylori infection and represents the main risk factor of gastric cancer ${ }^{1}$. Nowadays, inflammatory and preneoplastic lesions are underdiagnosed during gastric endoscopy under white light. There are usually no macroscopically observable differences between normal mucosa and pathological tissues at the stage of inflammatory and preneoplastic lesions, using classical white-light endoscopy and even using high-definition endoscopes ${ }^{2-5}$. To our knowledge, there is no technology capable of exploring large areas of gastric tissue during an endoscopic examination to detect gastritis. Confocal endomicroscopy and optical coherence tomography allow real-time analysis of tissue structure on specific lesions, but do not allow scanning of large mucous surfaces at the scale of an entire digestive organ. The diagnosis of gastritis is performed from the histological analysis of biopsies, randomly collected from gastric tissue during endoscopy. However, the collection of random biopsies increases the examination time and requires extra resources ${ }^{6}$. Therefore, identification of gastritis by an optical device could motivate the collection of biopsy samples by the endoscopist. Conversely, the collection of biopsies could be avoided in the absence of detectable inflammation.

The chemical structure and the architecture of pathological tissues induces modifications of light absorption and reflection ${ }^{7}$. As a result, abnormal tissues may reflect light with a different spectrum than normal mucosa. The reflectance corresponds to the ratio between the emitted and reflected light from the tissue. Multispectral imaging is capable of acquiring images on different wavelengths of the light spectrum and measuring reflectance. It has already been used in medical applications, mainly for the identification of skin lesions ${ }^{8,9}$. Therefore, a minimally invasive diagnosis based on multispectral imaging could highlight histological differences in gastric tissue to facilitate the detection of patients with gastritis. This would optimize the detection of gastritis in

${ }^{1}$ Department of Gastroenterology, Université Paris Saclay/UVSO, INSERM, Infection and Inflammation, UMR 1173, AP-HP, Hôpital Ambroise Paré, 9 avenue Charles de Gaulle, 92100 Boulogne Billancourt, France. 'Université Paris-Saclay, Institut d'Optique Graduate School, CNRS, Laboratoire Charles Fabry, 91127 Palaiseau, France. ${ }^{3}$ Université Paris-Saclay, UVSO, Inserm U1173, Infection et Inflammation, Laboratory of Excellence INFLAMEX, 78180 Montigny-Le-Bretonneux, France. ${ }^{4} \mathrm{ImViA}$ EA7535, Université Bourgogne Franche-Comté, Dijon, France. ${ }^{5}$ Department of Anatomical Pathology, Hôpital Ambroise Paré, AP-HP, 9 Avenue Charles de Gaulle, 92100 Boulogne-Billancourt, France. ${ }^{6}$ Institut Pasteur, Helicobacter Pathogenesis Unit, CNRS UMR 2001, 75724 Paris cedex 15, France. ${ }^{\circledR}$ email: thomas.bazin@aphp.fr 
patients, particularly those exhibiting inflammatory lesions with higher severity, leading to a better prevention of gastric cancer.

Using the mouse model of $H$. pylori infection to generate inflammatory gastric lesions and, in parallel, endoscopy examination in patients, the aim of our study was to determine the variation in reflectance of gastric tissue and its relationships with inflammatory lesions at different stages of gastritis.

\section{Methods}

Mouse model. Bacterial strains and growth conditions. The H. pylori strain SS1, able to colonize the mouse gastric mucosa for long periods ${ }^{10}$, was used in this study. Bacteria were grown on blood agar base 2 (Oxoid Lyon, France) plates supplemented with 10\% defibrinated horse blood (bioMérieux, Marcy L’Etoile, France) and an antibiotic-antifungal mixture. The plates were incubated at $37^{\circ} \mathrm{C}$ for $24-48 \mathrm{~h}$ under microaerobic conditions $\left(7 \% \mathrm{O}_{2}, 10 \% \mathrm{CO}_{2}\right.$; Anoxomat system).

Mouse infection. Mouse experiments were carried out in strict accordance with European recommendations. The protocol has been approved by the Committee of Central Animal Facility Board, the Ethic committee on animal experimentation of the Institut Pasteur (Ref 2013-0051) and the French Ministry of Higher Education and Research (Ref 00317.02).

Five-weeks old NMRI female mice (Charles River Laboratories; France) were housed in polycarbonate cages and acclimatized for 1 week before starting the experiments. In total 24 mice were included in the present study: $12 \mathrm{H}$. pylori SS1-infected mice, which were orogastrically inoculated with $150 \mu \mathrm{l}$ of a suspension of bacteria $\left(10^{8}\right.$ colony forming unit $(\mathrm{CFU}) / \mathrm{ml}$ ) and 12 non-infected mice which received $150 \mu \mathrm{l}$ of peptone broth. After $1,3,7$ and 12 months, 3 infected and 3 non-infected mice were sacrificed, their stomach isolated and fragments containing antrum and fundus parts were used for histology and reflectance spectra analysis of the gastric inflammatory lesions. In addition, H. pylori gastric colonization was quantified as previously described ${ }^{11}$.

Histological analysis. Stomach samples from non-infected and infected mice were fixed in RCL2 (Alphelys, France) and embedded in low-melting-point paraffin wax (Poly Ethylene Glycol Distearate; Sigma, USA). Four $\mu \mathrm{m}$-thick sections were stained by hematoxylin and eosin treatment (H\&E) and examined blindly for neutrophil infiltration and mononuclear cell infiltration, which were semi-quantitatively evaluated as previously described $^{12,13}$, based on a scoring system with four severity grades $(0=$ none, $1:$ mild, 2 : moderate, 3 : severe $)$ according to the Updated Sydney System ${ }^{14}$.

Tissue reflectance. The reflectance of the mucosa was measured using two different methods: (a) a spectrometer Konica Minolta CM-2600d, which has an integrated source of light and retrieves the reflectance of the measured surface (6 mm diameter disk) from 400 to $740 \mathrm{~nm}$, at $10 \mathrm{~nm}$ increments (Fig. 3A); (b) a multispectral camera Flux Data FD1665, retrieving 7 monoband images from 480 to $810 \mathrm{~nm}$ (width from 30 to $100 \mathrm{~nm}$ ), from which 6 are in the visible spectrum and 1 in the near infrared. This camera has 3 independent sensors for the acquisition. The acquisition surface was also $6 \mathrm{~mm}$ disks. Acquisitions were performed using a dedicated software from the University of Bourgogne. The light source was a Xenon lamp. In order to reduce the specularity, an angle of $45^{\circ}$ was configured between the light source and the multispectral camera. In addition, two linear polarizers were included, one for the light source and the other for the multispectral camera configured in cross polarization. A white calibration was performed before each set of acquisition.

Human. Subjects. We included patients referred to the endoscopic unit of Ambroise Paré hospital, Boulogne-Billancourt, France. Patients were eligible if they had been scheduled for gastroendoscopy under general anesthesia and had given their written informed consent, as approved by the Comite de Protection des Personnes Sud-Est III ethics committee on June 2019 (registration number: 19.06.21.76520). The study was conducted according to the World Medical Association Declaration of Helsinki. Patients with chronic gastritis $(n=8)$ were enrolled from the cohort GASTRIMED (ClinicalTrials.gov identifier: NCT02325323) funded by the French Gastroenterology Society (SNFGE), including adults which had a gastroscopy in the last 6 months with antral and gastric body biopsies. The aim of this cohort is to study the association between changes in the gastric mucosa in chronic gastritis and the onset of cancer.

Procedure. Multispectral acquisition. During gastroendoscopic examination, the endoscope was focused on the antrum. Then we used the multispectral acquisition system as described in ${ }^{15}$. A xenon lamp was used as the light source, since it provides constant stable illumination, which can be easily filtered in order to provide illumination at different wavelengths. In order to acquire multispectral images, the light was filtered by a rotating filter wheel. Thanks to the sequential rotation of filters, the tissue was illuminated with a specific wavelength for a short period of time. The filter wheel contained 5 filters centered at $440 \mathrm{~nm}, 520 \mathrm{~nm}, 560 \mathrm{~nm}, 600 \mathrm{~nm}$ and $640 \mathrm{~nm}$, with a full width at half maximum of $80 \mathrm{~nm}$. The acquisition time for one full rotation of the filter wheel was slightly more than one second. At the same time, images were collected by the native camera from the gastroendoscope (Olympus Exera II) with the magnification option disabled.

This device allowed us to capture a multispectral image from the antrum after being illuminated sequentially with 5 different wavelengths. The time allocated for the collection of multispectral images was $30 \mathrm{~s}$, which was enough to perform multiple acquisitions. 
Biopsy collection. After multispectral acquisition we switched to the normal white light examination mode in order to perform systematic biopsy collection from the antrum and the corpus, according to standard protocol. These biopsies were placed in $10 \%$ formalin and embedded in paraffin. Transversal gastric tissue section samples were stained by H\&E for routine histological analysis. In addition, polymerase chain reaction (PCR) and immunochemistry using PFLEX Polyclonal Rabbit antibody (Dako) were performed to detect H. pylori infection. Biopsies were scored for the severity of inflammatory lesions according to the Updated Sydney System ${ }^{14}$. The histopathological diagnosis was performed by a senior pathologist (CJ).

Image and spectral treatment. Each gastroendoscopic video, acquired during endoscopy procedure, was divided in sequences of multispectral images. Each multispectral image contained 5 images $(640 \times 480$ pixels, 8 bits), one for each wavelength used for illumination.

The gastric wall could perform some movements during the acquisition. In order to correct these micromovements of the tissue, we used multispectral images where the images at each wavelength were sharp and showed low movements between them. Next, the registered multispectral images were thresholded in order to remove specular areas. In addition, the user manually selected areas of tissue from the image which were correctly exposed in order to remove artifacts such as bubbles of gastric juice, borders of folds of gastric tissue and the pylorus sphincter. Then, spectra were standardized by mathematical normalization to avoid the influence of the distance and angle between the tissue and the gastroendoscope.

Statistical analysis. The spectra from the multispectral images were randomly sampled from the observed areas of gastric tissue. The sample consists of 20 areas of the image of size $5 \times 5$ pixels from each multispectral image, which were averaged in order to retrieve a representative spectrum for each multispectral image. This procedure was repeated 10 times for each multispectral image.

After separation into two clinical groups (normal $v s$ gastritis) based on histology analysis, we computed a reference spectrum for the normalized data of the control group, as the median response at each captured wavelength. Then, each patient from the gastritis group was compared to the control group in order to measure the percentage of variation.

In order to compare the differences between the spectra from the two groups, Wilcoxon-Mann-Whitney test was performed with Bonferroni correction (posthoc) on the normalized spectra; $\mathrm{p}$ values $<0.05$ were considered statistically significant.

Raw data are available on request.

Ethical approval. This study was approved by the Comité de Protection des Personnes Sud-Est III ethics committee in June 2019 (registration number: 19.06.21.76520). A written informed consent was obtained from all the patients involved in the study.

\section{Results}

Mouse. Histological analysis. The gastric mucosa of all infected mice was efficiently colonized by the $H$. $p y$ lori strain SS1 at each time-point. Mean of CFU per gram of gastric tissue \pm SD was $2 \pm 3,0.8 \pm 1.5,0.2 \pm 0.5,0.2 \pm$ 20 at month (M)1, M3, M7 and M12, respectively. No H. pylori colonization was observed in the control groups. Histological modifications were similar to those observed in previously published work ${ }^{16}$. After 1 month of infection (M1), gastric histological analysis showed low mononuclear cell infiltration without any polynuclear cell infiltrate [total score $0.5 \pm 0.1$ (mean \pm SD)]. At M3 a dense polynuclear infiltrate was observed, corresponding to a mild inflammation (total score $4.5 \pm 0.5$ ). At M7 and M12 the infiltrate was only mononuclear, corresponding to a moderate (total score 3.2 \pm 0.3 ) and mild (total score $2.1 \pm 0.3$ ) chronic inflammation at M7 and M12, respectively (Figs. 1b, 2). No histological inflammation was found in the control groups (Fig. 1a). Histological images at M12 can be found online as Supplementary Figures S1-S6 for infected mice at M12, and Supplementary Figure S7 for non-infected mice at M12.

Spectrometer. The acquisition spectra of the infected stomachs at each time-points, normalized to the corresponding control stomachs are presented in Fig. 3B. Of note, reflectance variations were not significant in controls. We identified 3 bands of wavelengths which were significantly modified between the two groups at more than one time-point. These bands correspond to $430-450 \mathrm{~nm}, 470-590 \mathrm{~nm}$ and $620-660 \mathrm{~nm}$. In the infected groups, the reflectance band between 430 and $450 \mathrm{~nm}$ was significantly reduced at M1 and M3. The reflectance band between 470 and $590 \mathrm{~nm}$ was significantly reduced at M3 and M7 and increased at M12. In addition, the reflectance band between 620 and $660 \mathrm{~nm}$ was significantly increased at M1 and reduced at M3. Of note, spectrum intensity was significantly reduced at all wavelengths at M3.

Multispectral camera. Results are presented in Fig. 4. Again, reflectance variations were not significant in controls. The reflectance band between 420 and $460 \mathrm{~nm}$ was reduced at M7 and M12. The reflectance band between 510 and $540 \mathrm{~nm}$ was reduced at M7. The reflectance band between 540 and $590 \mathrm{~nm}$ was reduced at M7. The reflectance band between 590 and $620 \mathrm{~nm}$ was increased at M1. The reflectance band between 620 and $720 \mathrm{~nm}$ was increased at M1 and reduced at M7. The reflectance band between 720 and $810 \mathrm{~nm}$ was reduced at M7.

Whatever the reflectance acquisition method used, no correlation was found between the level of $H$. pylori gastric colonization, the score grading of the inflammatory lesions and the intensity of the spectra observed. 


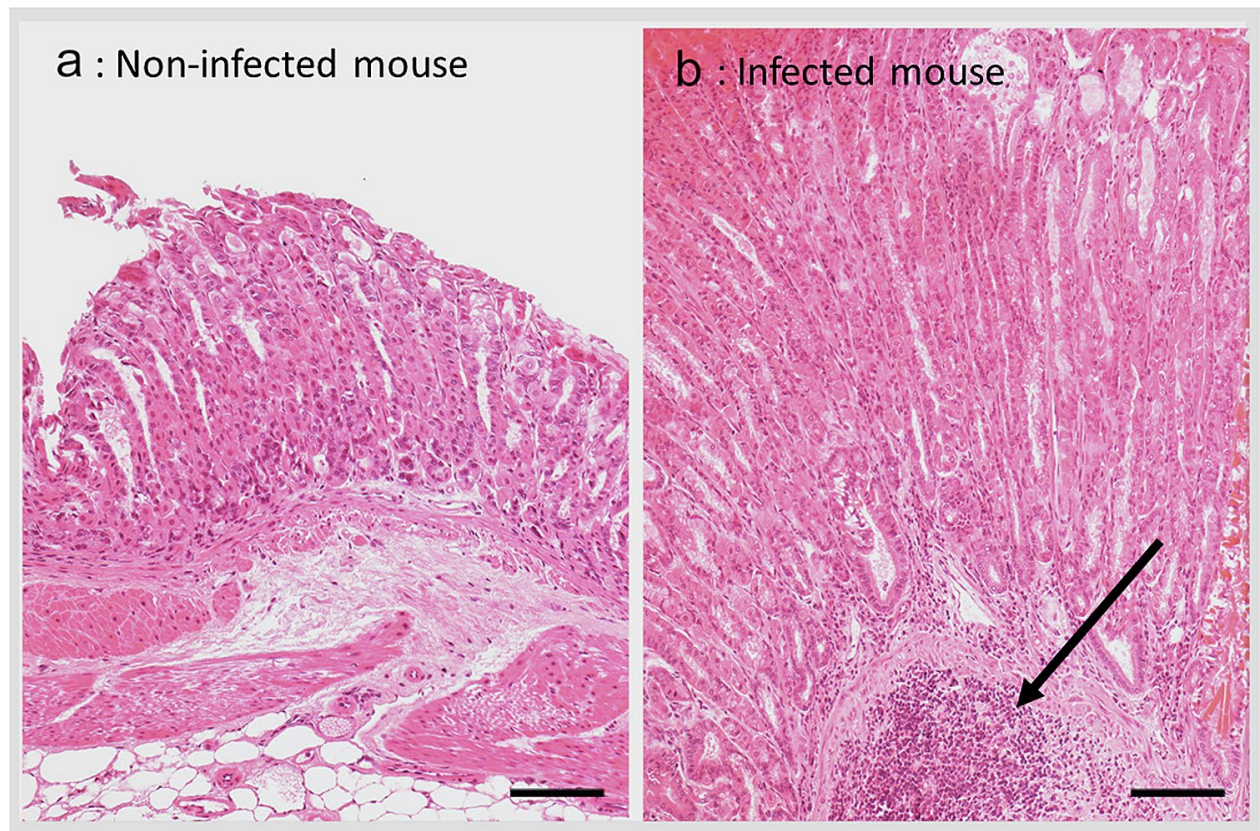

Figure 1. Gastric histology at 12 months in non-infected control $(\mathrm{n}=12$, a $)$ and $H$. pylori infected mice $(\mathrm{n}=12$, b). Mononuclear cell infiltration and aggregates (arrow) are easily visible in the infected mucosa (scale bar $100 \mu \mathrm{m})$ (Photo L. Fiette, Institut Pasteur).

6

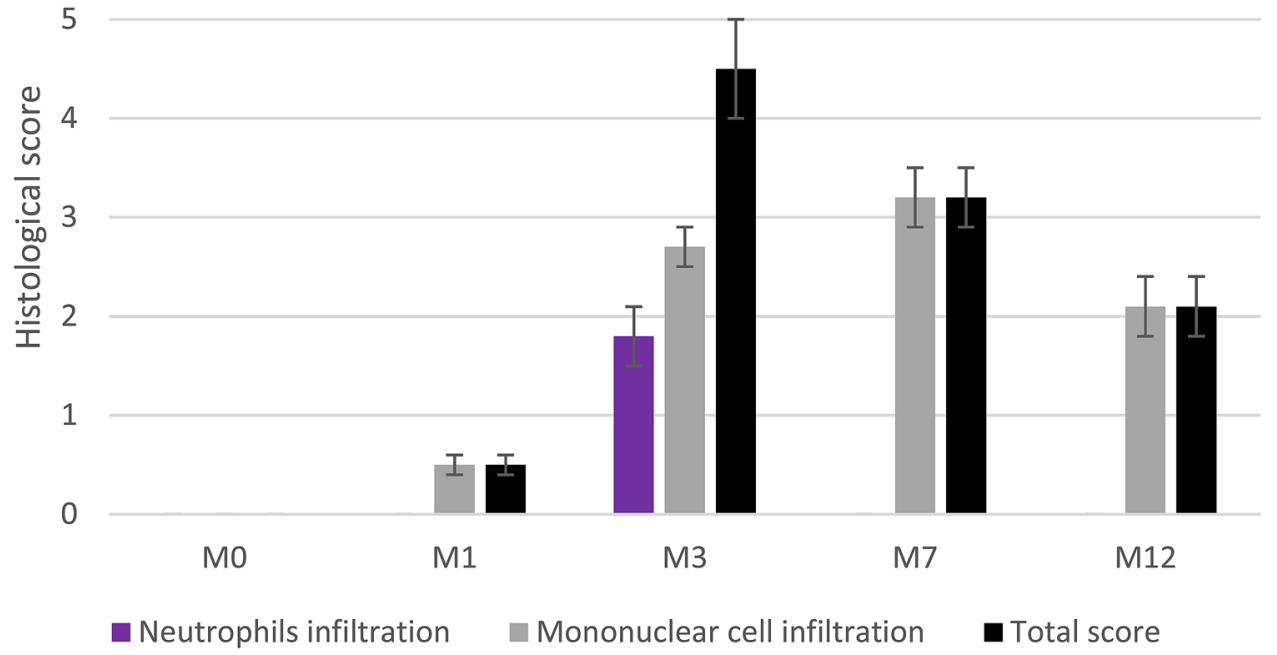

Figure 2. Evolution of mouse gastric mucosa inflammation after H. pylori infection. Values are means of histological score \pm SD.

Human. Clinical and pathological characteristics of patients. The present study was performed over a period of one year between July 2019 and February 2020. A written informed consent was obtained from all the patients involved in the study. We collected a total of 62 gastroendoscopic videos from 62 patients included in GASTRIMED cohort. We only kept 25 videos which did not show any evidence of visible lesions under white light, in order to avoid re-emitted light modification due to macroscopic abnormalities such as gastric atrophy or ulcers. The patients were grouped according to one of the two clinical conditions based on histological results: control group or gastritis. The control group presented only rare mononuclear cells in the mucosa $($ Sydney score $=0)$ and no $H$. pylori infection. On the other hand, the pathological group were positive for $H$. pylori infection, as assessed by immunohistochemistry or by specific PCR, and inflammatory infiltrate including mononuclear and 


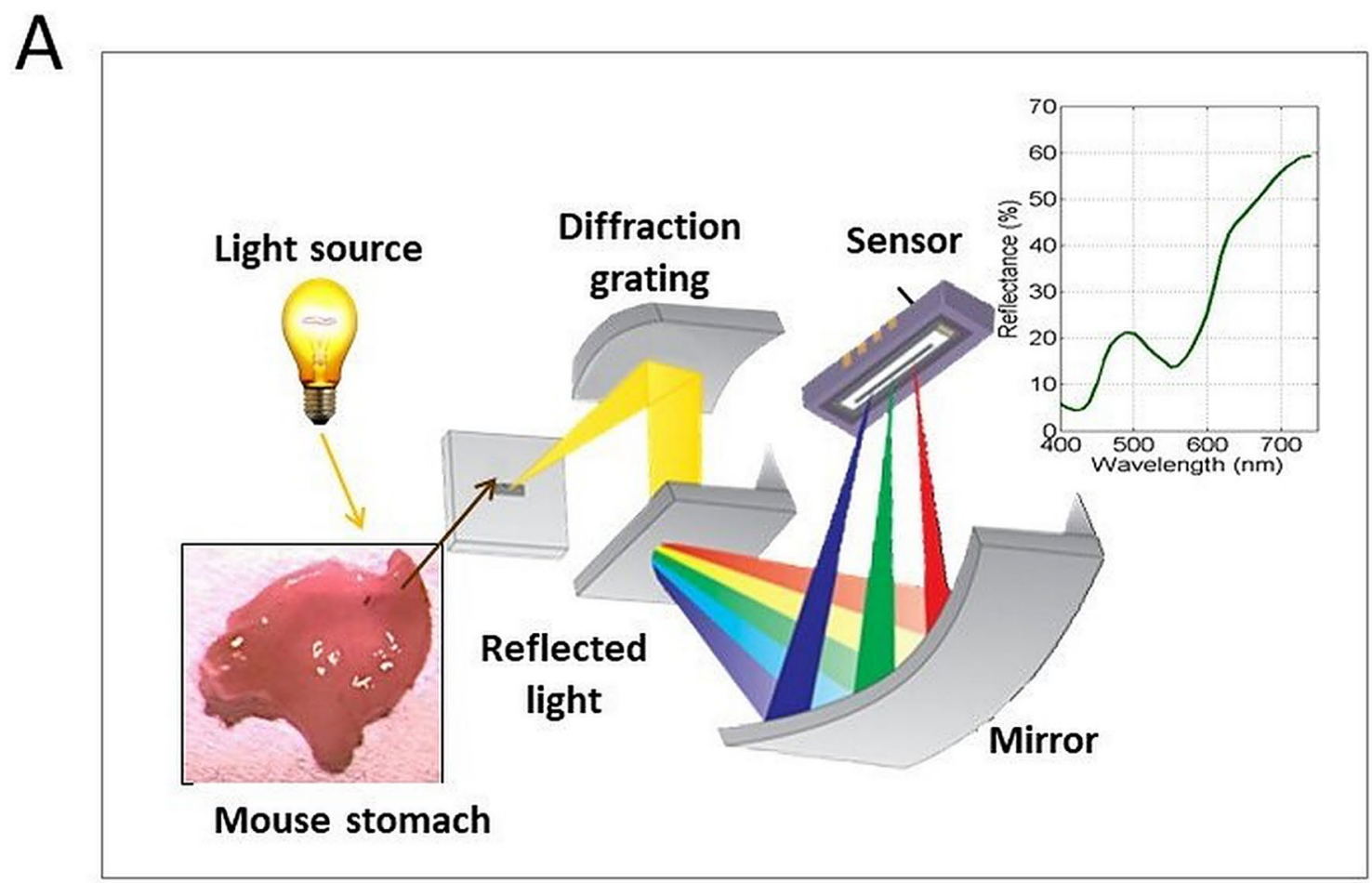

B

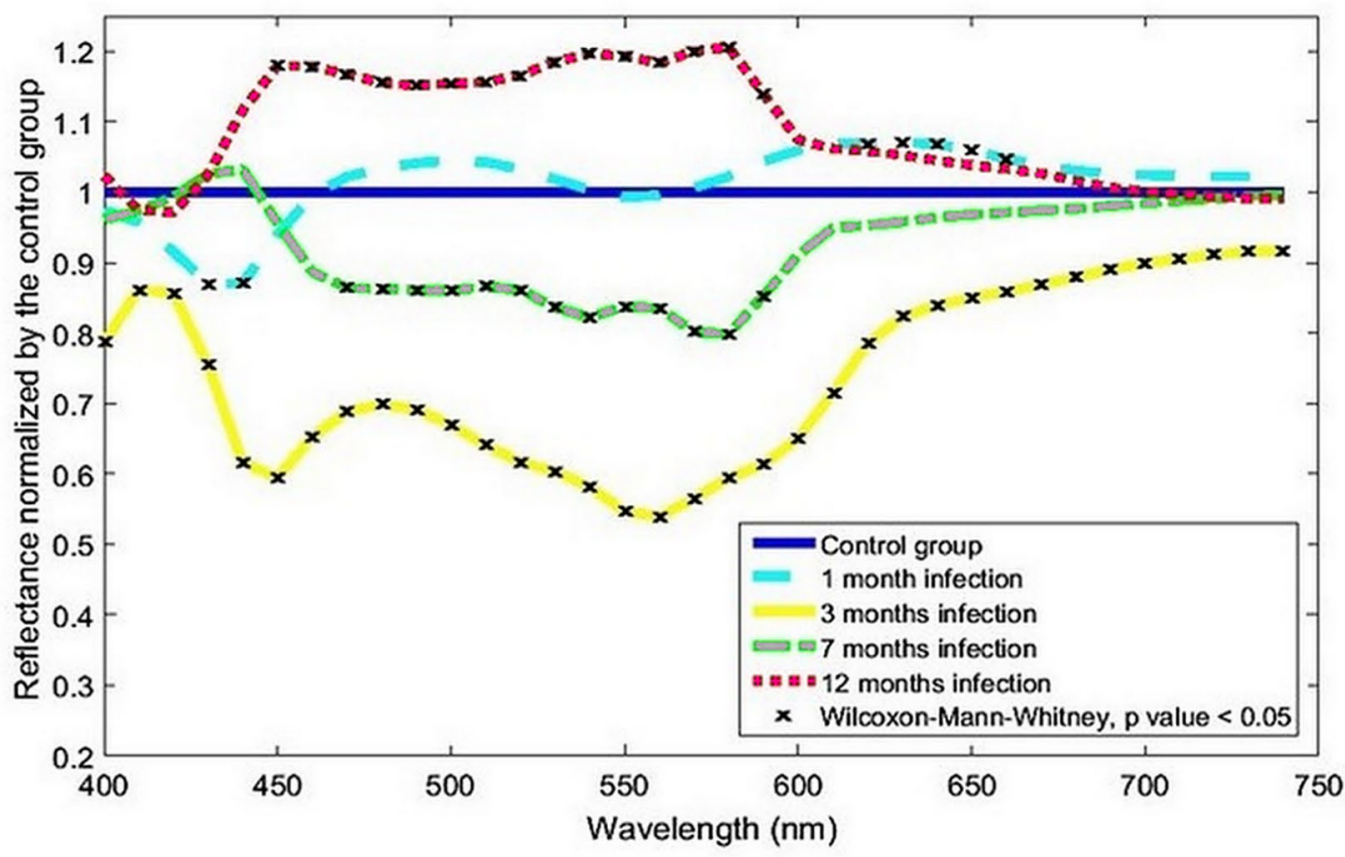

Figure 3. (A) Spectrometer setup and principle. The light reflected from the tissue is decomposed and measured all along the visual light spectrum. (B) Reflectance variations, normalized by the control group, measured using the spectrometer, at each time point (10 $\mathrm{nm}$ increment).

polynuclear cells [Sydney score $=1.1 \pm 1.0($ mean \pm SD)]. In Table 1 are presented the clinical features of the two groups. There were no significant differences in gender or age between the groups.

Multispectral analysis. The mean number of images studied was $4.6( \pm 1.1)$ in gastritis group and $5.3( \pm 0.9)$ in control group (mean \pm SEM, NS). The mean pixel number analyzed was 23,125 ( \pm 5664$)$ in the gastritis group 


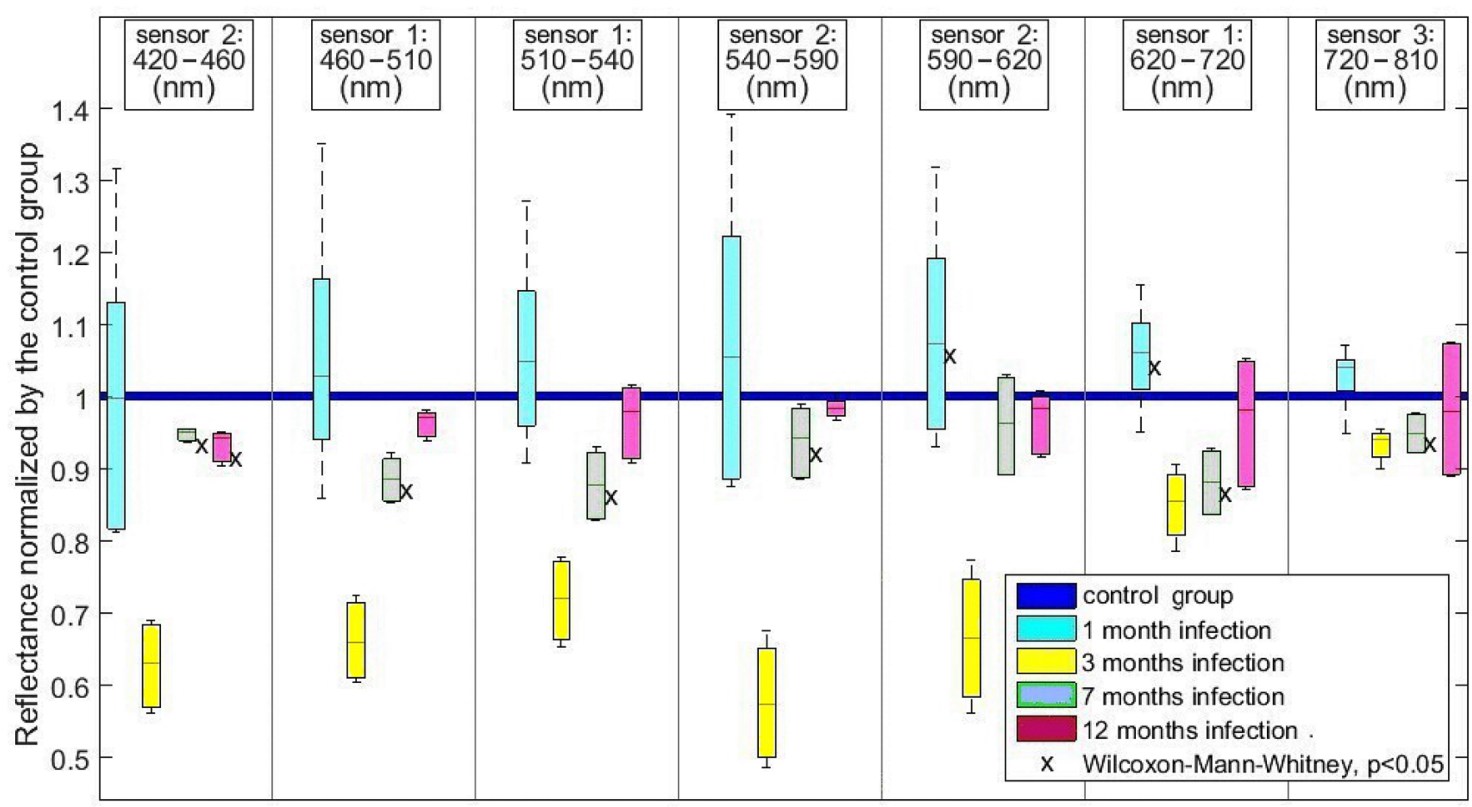

Figure 4. Reflectance variations obtained using the multispectral camera and normalized on the non-infected control group, for each monoband image and at each time-point of $H$. pylori infection.

\begin{tabular}{|l|l|l|l|}
\hline & N & Age $( \pm$ SEM) & Gender ratio $($ H:F $)$ \\
\hline Gastritis group & 8 & $54.9( \pm 5.8)$ & $3: 1$ \\
\hline Control group & 17 & $59.6( \pm 2.6)$ & $0.7: 1$ \\
\hline
\end{tabular}

Table 1. Patients characteristics.

and 26,470 ( \pm 4613$)$ in the control group (mean \pm SEM, NS) (see Method section, Image and spectral treatment sub-section for the description of image pre-processing).

The spectra analysis in Fig. 5 showed that the intensity of wavelengths at $560 \mathrm{~nm}, 600 \mathrm{~nm}$ and $640 \mathrm{~nm}$ was statistically different for active gastritis patients, compared to the normal group.

The wavelength of $560 \mathrm{~nm}$ showed a significant decrement of the reflected light with respect to the reference of the control group. On the other hand, we observed a significant increment of the reflected light at the wavelengths of $600 \mathrm{~nm}$ and $640 \mathrm{~nm}$ in the gastritis group.

No correlation was found between reflectance variation and Sydney scores.

The multispectral images were observed by senior endoscopists (DL and TB). As it was stated in the protocol, we were not able to observe differences between images under white light from control or gastritis patients, as illustrated in Fig. 6a,b. Consequently, the differences observed were only due to illumination with specific wavelengths. The band at $560 \mathrm{~nm}$ enhanced the contrast and highlighted areas with a wavy appearance in patients with active inflammation compared to controls as shown in Fig. 6c,d. This showed evidence of texture modification which was not visible under white light. In contrast, the images from subjects in the control group showed a smooth uniform mucosal surface.

\section{Discussion}

To our knowledge, the present study is the first that analyzes in parallel the reflected light properties of gastric tissue inflammation related to $H$. pylori infection in the mouse model, and multispectral in vivo images of the stomach in humans.

In mouse, the variations in reflectance differ according to the timepoints: the values generally tend to increase at M1 (and at M12 for the spectrometer), and to decrease at other timepoints. We do not have data to precisely explain these variations, but the differences in histological sub-scores could be an explanation. For example, neutrophil infiltration could explain the increase in reflectance at M3. Similarly, at 12 months, the large aggregates of inflammatory cells observed could explain the change in reflectance.

Our data from the spectrometer and multispectral camera show statistically significant changes in reflectance at different stages of the inflammatory lesions induced by $H$. pylori in the gastric mucosa of mice infected from 1 to 12 months. Importantly, the sensitivity of reflectance analysis is such that it can measure reflectance variations even only 1 month after $H$. pylori infection, where no inflammatory lesions are identified by histological analysis (Sydney score less than one). Tissue inflammation is not limited to cellular infiltrate. Other phenomena such as 


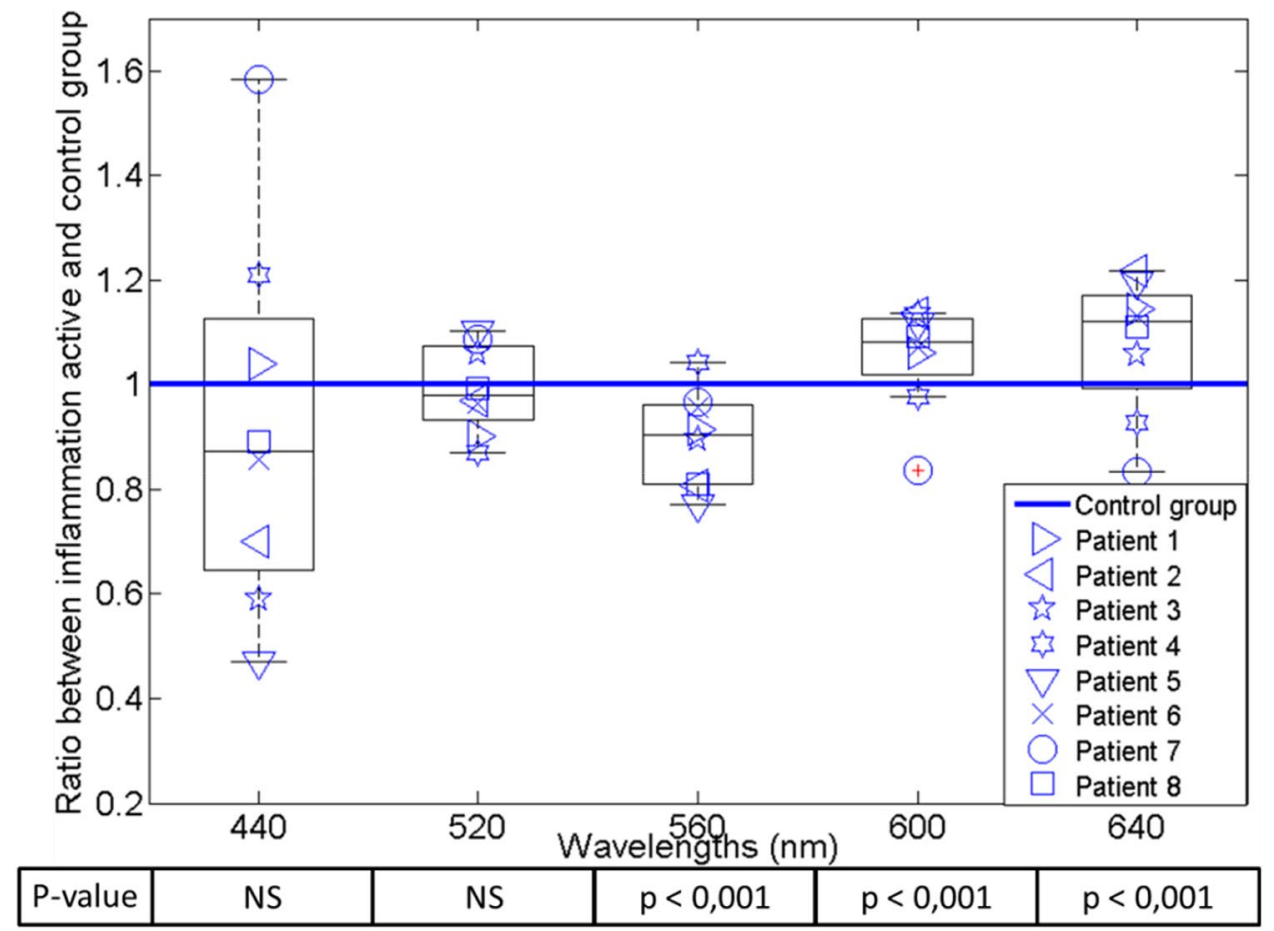

Figure 5. Ratio of variation in the spectrum from patients with active inflammation compared with the reference spectrum from the control group. P values were obtained using Wilcoxon-Mann-Whitney test.

hemoglobin saturation, water concentration levels and cytoskeleton rearrangements are involved in inflammatory process and can play a role in reflectance modifications ${ }^{17}$.

In mice, whatever the method of light analysis used, we observed that the reflectance between 620 and $660 \mathrm{~nm}$ was statistically different after 1 and 3 months of infection. The changes at these wavelengths could be explained by differences in blood oxygenation, as oxyhemoglobin reflectance is higher between 600 and $700 \mathrm{~nm}$ in comparison with deoxy-hemoglobin ${ }^{18}$. At 12 months, the reflectance between 450 and $590 \mathrm{~nm}$ was significantly different between the infected and non-infected groups; these modifications could be related to size changes of the scatters and again the oxygen saturation of the tissue ${ }^{19}$.

The resulting tendency between the two methods in mice is identical (the variations go in the same direction), except at M12, where the spectrometer values are globally higher than in the control group, and the multispectral camera values are globally lower. This could be related to the uneven spatial distribution of lymphocyte aggregates which is only observable at M12. As reflectance measurements are based on $6 \mathrm{~mm}$ disks, they could simply miss lymphocytes aggregates.

Spectrometer is the gold standard of light intensity measurement, with small increments and high sensitivity. The spectral resolution of the multispectral camera is significantly lower than that of the spectrometer. But camera spatial resolution is higher, and its wide range of acquisition allows the analysis of near infrared wavebands, unlike the spectrometer we used. Aiming to extrapolate our results to endoscopic methods for surveillance of large surfaces of tissues, results from the multispectral camera are of peculiar interest, especially since they are coherent with those of the spectrometer. Of note, multispectral camera results in mice did not show any statistical significance at M3 despite high differences, probably due to high variability.

In humans, changes in light based on tissue properties have been shown to be useful for diagnosis in human health ${ }^{7,20}$. New technology, such as narrow band imaging (NBI, Olympus), takes advantage of the tissue response at different wavelengths. It provides qualitative images that show clues of the sub-epithelial capillary network, by measuring the peaks of absorption of hemoglobin. However, the inflammatory cell infiltration cannot be detected as there is no significant change in the endoscopic appearance during the gastritis condition. In contrast with these systems, the optical device that we used during patients endoscopies analyzed more wavelengths and could be used for diagnosis by identifying inflammation-related quantitative changes in the tissue.

In humans, we observed that reflectance wavelengths at $560 \mathrm{~nm}, 600 \mathrm{~nm}$ and $640 \mathrm{~nm}$ are different in gastritis patients. Areas with a wavy appearance were highlighted at $560 \mathrm{~nm}$; this wavelength is related to the absorption peak of hemoglobin and correlated to micro vessels density ${ }^{21,22}$. The reflectance changes observed at $600 \mathrm{~nm}$ and $640 \mathrm{~nm}$ could be linked with variation of cytoskeleton components, as collagen networks involved in tissue repair may also change the reflectance above $600 \mathrm{~nm}^{23-26}$. It should be noted that we observed a similar decrease of reflectance at $560 \mathrm{~nm}$ between infected mice at M7 and patients with gastritis. This observation suggests that similar events occurring both in mice and humans could account for the observed spectra. However, the identification of the physiological and biochemical origin of the mechanisms which, during inflammation, are responsible for the variation in reflectance need to be further investigated. 

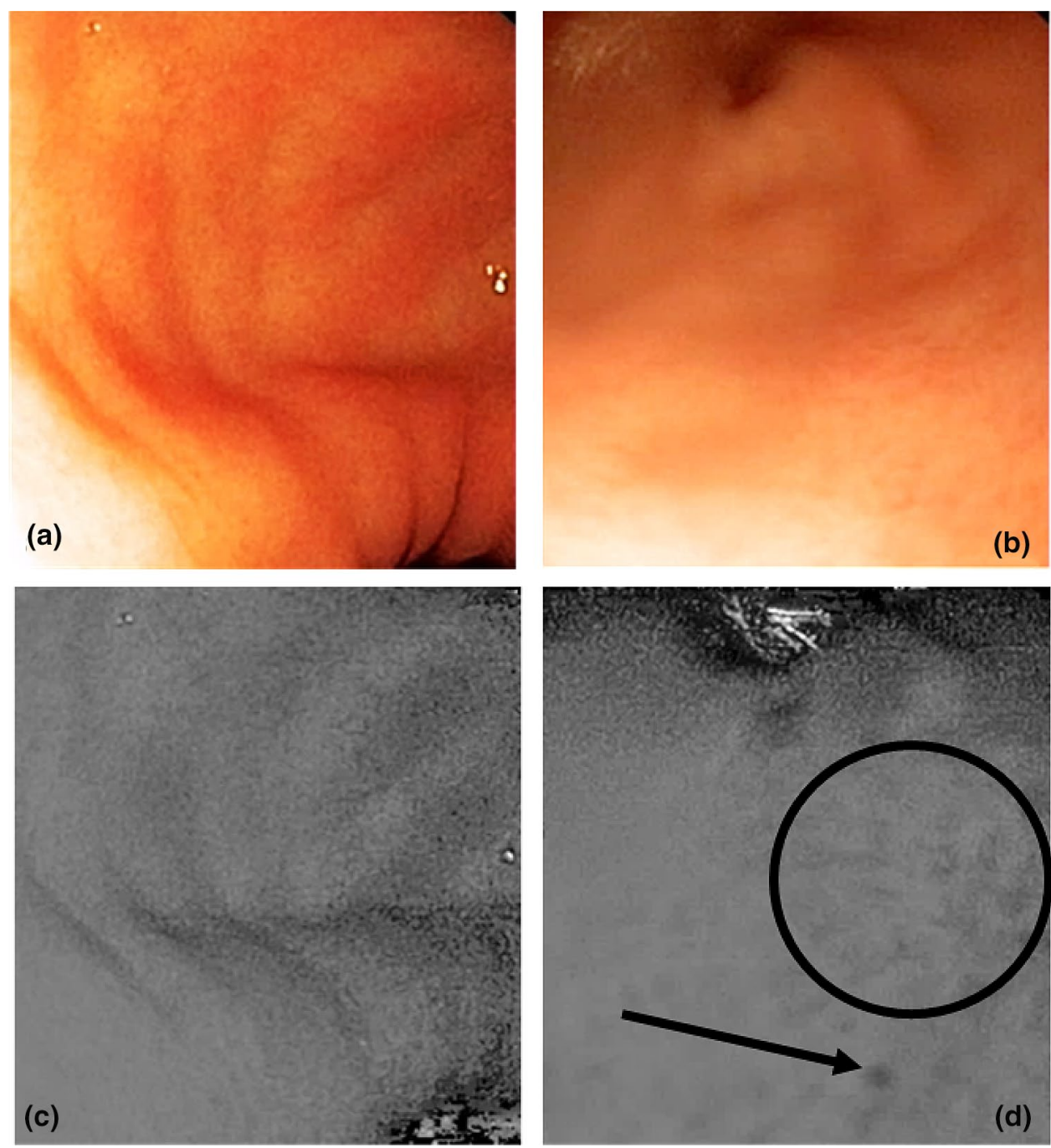

Figure 6. Images under white light from the control group (a) and gastritis patients (b). In the band at $560 \mathrm{~nm}$, we observed that the tissue from the control group (c) was smooth, compared to the gastritis tissue (d) which presented black spots (arrow) and a non-uniform surface. The band at $560 \mathrm{~nm}$ highlighted areas with a wavy appearance (black circle) in the patients with inflammation (d).

Interestingly, our method does not require any external marker, unlike other techniques using a marker that is injected or applied locally ${ }^{27}$. The safety and accessibility of this method are therefore guaranteed.

In this exploratory study, using multispectral imaging, we have robustly shown in the mouse model and in humans that gastritis mucosa exhibits modifications of reemitted light around $560 \mathrm{~nm}, 600 \mathrm{~nm}$ and $640 \mathrm{~nm}$. These promising results pave the way for the development of improved endoscopes with tailored virtual chromoendoscopic properties to detect gastritis. These three wavelengths could thus be used as biomarkers of gastritis and allow real-time optical biopsy obtention, leading to a better prevention of gastric cancer with cost efficient endoscopies.

Received: 18 April 2020; Accepted: 2 November 2020

Published online: 18 November 2020

\section{References}

1. Correa, P. \& Piazuelo, M. B. The gastric precancerous cascade. J. Dig. Dis. 13, 2-9 (2012).

2. Bah, A. et al. Endoscopic features of Helicobacter pylori-related gastritis. Endoscopy 27, 593-596 (1995).

3. Redéen, S., Petersson, F., Jönsson, K.-A. \& Borch, K. Relationship of gastroscopic features to histological findings in gastritis and Helicobacter pylori infection in a general population sample. Endoscopy 35, 946-950 (2003).

4. Laine, L., Cohen, H., Sloane, R., Marin-Sorensen, M. \& Weinstein, W. M. Interobserver agreement and predictive value of endoscopic findings for H. pylori and gastritis in normal volunteers. Gastrointest. Endosc. 42, 420-423 (1995).

5. Calabrese, C. et al. Correlation between endoscopic features of gastric antrum, histology and Helicobacter pylori infection in adults. Ital. J. Gastroenterol. Hepatol. 31, 359-365 (1999). 
6. Gonen, C., Simsek, I., Sarioglu, S. \& Akpinar, H. Comparison of high resolution magnifying endoscopy and standard videoendoscopy for the diagnosis of Helicobacter pylori gastritis in routine clinical practice: A prospective study. Helicobacter 14, 12-21 (2009).

7. Bigio, I. \& Mourant, J. Optical Biopsy. in Encyclopedia of Optical and Photonic Engineering (eds. Driggers, R. \& Hoffman, C.) (CRC Press, 2014).

8. Tomatis, S. et al. Automated melanoma detection with a novel multispectral imaging system: Results of a prospective study. Phys. Med. Biol. 50, 1675-1687 (2005).

9. Jolivot, R., Benezeth, Y. \& Marzani, F. Skin parameter map retrieval from a dedicated multispectral imaging system applied to dermatology/cosmetology. Int. J. Biomed. Imaging 2013, 978289 (2013).

10. Lee, A. et al. A standardized mouse model of Helicobacter pylori infection: Introducing the Sydney strain. Gastroenterology 112, 1386-1397 (1997).

11. Ferrero, R. L., Cussac, V., Courcoux, P. \& Labigne, A. Construction of isogenic urease-negative mutants of Helicobacter pylori by allelic exchange. J. Bacteriol. 174, 4212-4217 (1992).

12. Prophet, E. B. \& (U.S.), A. F. I. of P. Laboratory Methods in Histotechnology. (American Registry of Pathology, 1992).

13. Eaton, K. A., Radin, M. J. \& Krakowka, S. An animal model of gastric ulcer due to bacterial gastritis in mice. Vet. Pathol. Online 32, 489-497 (1995).

14. Dixon, M. F., Genta, R. M., Yardley, J. H. \& Correa, P. Classification and grading of gastritis. The updated Sydney System. Int. Workshop Histopathol. Gastritis Houst. 20, 1161-1181 (1994)

15. Martinez-Herrera, S. E. et al. Identification of precancerous lesions by multispectral gastroendoscopy. Signal Image Video Process. 10, 455-462 (2016).

16. Touati, E. et al. Chronic Helicobacter pylori infections induce gastric mutations in mice. Gastroenterology 124, 1408-1419 (2003).

17. Phillips, K. G. et al. Dermal reflectivity determined by optical coherence tomography is an indicator of epidermal hyperplasia and dermal edema within inflamed skin. J. Biomed. Opt. 16, 040503 (2011).

18. Bender, J. E. et al. Noninvasive monitoring of tissue hemoglobin using UV-VIS diffuse reflectance spectroscopy: A pilot study. Opt. Express 17, 23396-23409 (2009).

19. Marois, M., Jacques, S. L. \& Paulsen, K. D. Optimal wavelength selection for optical spectroscopy of hemoglobin and water within a simulated light-scattering tissue. J. Biomed. Opt. 23, 1-5 (2018).

20. Ortega, S., Fabelo, H., Iakovidis, D. K., Koulaouzidis, A. \& Callico, G. M. Use of hyperspectral/multispectral imaging in gastroenterology. Shedding some-different-light into the dark. J. Clin. Med. 8, 20 (2019).

21. Yao, K., Yao, T., Matsui, T., Iwashita, A. \& Oishi, T. Hemoglobin content in intramucosal gastric carcinoma as a marker of histologic differentiation: A clinical application of quantitative electronic endoscopy. Gastrointest. Endosc. 52, 241-245 (2000).

22. Goto, A. et al. Use of hyperspectral imaging technology to develop a diagnostic support system for gastric cancer. J. Biomed. Opt. 20, 016017 (2015).

23. Filer, A., Raza, K., Salmon, M. \& Buckley, C. D. Targeting stromal cells in chronic inflammation. Discov. Med. 7, 20-26 (2007).

24. Naylor, A. J., Filer, A. \& Buckley, C. D. The role of stromal cells in the persistence of chronic inflammation. Clin. Exp. Immunol. 171, 30-35 (2013).

25. McGettrick, H. M., Butler, L. M., Buckley, C. D., Rainger, G. E. \& Nash, G. B. Tissue stroma as a regulator of leukocyte recruitment in inflammation. J. Leukoc. Biol. 91, 385-400 (2012).

26. Chandra, M. et al. Quantitative molecular sensing in biological tissues: An approach to non-invasive optical characterization. Opt. Express 14, 6157-6171 (2006).

27. Kossatz, S. et al. Validation of the use of a fluorescent PARP1 inhibitor for the detection of oral, oropharyngeal and oesophageal epithelial cancers. Nat. Biomed. Eng. 4, 272-285 (2020).

\section{Acknowledgements}

We thank Laurence Fiette (Institut Pasteur Institut Mutualiste Montsouris, Paris, France) for her help with the histological analysis and Richard Wheeler (Institut Pasteur, Paris, France) for reading the manuscript.

\section{Author contributions}

T.B., S.E.M.H. and D.L. drafted the work. S.E.M.H., A.J.M., C.J., J.F.E., V.M. acquired data. T.B., S.E.M.H., Y.B., E.T., F.M., D.L., M.B., F.G. interpreted data. T.B., S.E.M.H., Y.B., M.B., C.J., J.F.E., F.G., E.T., F.M., D.L. designed the work. Y.B., E.T., F.M., M.B., F.G. substantively revised the work. All authors reviewed the manuscript.

\section{Funding}

This work was supported by the Initiatives d'Excellence (IDEX) Paris-Saclay, France (Grant to SEMH), the Conseil Régional de Bourgogne, France (FM), the Fond Européen de Développement Régional FEDER, the Institut Pasteur through the donation from the Odyssey-Reinsurance Company (ET) and the French Gastroenterology Society (SNFGE) that funded the GASTRIMED cohort (DL).

\section{Competing interests}

The authors declare no competing interests.

\section{Additional information}

Supplementary information is available for this paper at https://doi.org/10.1038/s41598-020-77145-4.

Correspondence and requests for materials should be addressed to T.B.

Reprints and permissions information is available at www.nature.com/reprints.

Publisher's note Springer Nature remains neutral with regard to jurisdictional claims in published maps and institutional affiliations. 
(c) (i) Open Access This article is licensed under a Creative Commons Attribution 4.0 International cc) License, which permits use, sharing, adaptation, distribution and reproduction in any medium or format, as long as you give appropriate credit to the original author(s) and the source, provide a link to the Creative Commons licence, and indicate if changes were made. The images or other third party material in this article are included in the article's Creative Commons licence, unless indicated otherwise in a credit line to the material. If material is not included in the article's Creative Commons licence and your intended use is not permitted by statutory regulation or exceeds the permitted use, you will need to obtain permission directly from the copyright holder. To view a copy of this licence, visit http://creativecommons.org/licenses/by/4.0/.

(C) The Author(s) 2020, corrected publication 2021 